\title{
ARTICLE
}

\section{Juxtacrine interaction of macrophages and bone marrow stromal cells induce interleukin-6 signals and promote cell migration}

\author{
Jia Chang ${ }^{1,2}$, Amy J Koh ${ }^{1}$, Hernan Roca ${ }^{1}$, and Laurie K McCauley ${ }^{1,3}$
}

The bone marrow contains a heterogeneous milieu of cells, including macrophages, which are key cellular mediators for resolving infection and inflammation. Macrophages are most well known for their ability to phagocytose foreign bodies or apoptotic cells to maintain homeostasis; however, little is known about their function in the bone microenvironment. In the current study, we investigated the in vitro interaction of murine macrophages and bone marrow stromal cells (BMSCs), with focus on the juxtacrine induction of IL-6 signaling and the resultant effect on BMSC migration and growth. The juxtacrine interaction of primary mouse macrophages and BMSCs activated IL-6 signaling in the co-cultures, which subsequently enhanced BMSC migration and increased BMSC numbers. BMSCs and macrophages harvested from IL-6 knockout mice revealed that IL-6 signaling was essential for enhancement of BMSC migration and increased BMSC numbers via juxtacrine interactions. BMSCs were the main contributor of IL-6 signaling, and hence activation of the IL-6/ gp130/STAT3 pathway. Meanwhile, macrophage derived IL-6 remained important for the overall production of IL-6 protein in the co-cultures. Taken together, these findings show the function of macrophages as co-inducers of migration and growth of BMSCs, which could directly influence bone formation and turnover.

Bone Research (2015) 3, 15014; doi:10.1038/boneres.2015.14; Published online: 16 June 2015

\section{INTRODUCTION}

Mesenchymal stem cells (MSCs) and macrophages are cell populations that play very important roles in maintaining homeostasis of the bone marrow environment. MSCs are multipotent cells that differentiate into osteoblasts and form bone. 'Macrophages contribute to the innate immune response by eliminating bacteria, viruses, and clearing apoptotic bodies. Certain distinct macrophage subpopulations located in the brain (microglia), eye, and testes are believed to play key roles in tissue-specific remodeling and homeostasis. $^{2}$ In the bone marrow, macrophages share a common lineage with osteoclasts which are responsible for bone resorption and turnover. Meanwhile, they phagocytose apoptotic cells such as osteoblasts and BMSCs in order to maintain homeostasis of the bone marrow environment. ${ }^{3}$

Recently, a new role for macrophages has emerged and is under investigation. This role centers on macrophage function in the maintenance of bone marrow homeostasis during bone fracture healing. ${ }^{4}$ Macrophages are one of the earliest and most abundant cells in the bone environment after injury and are instrumental for normal bone healing by clearing local apoptotic cells and subsequently signaling to initiate bone formation. ${ }^{5-6}$ Interestingly, when macrophages were depleted from the healing sites, there was significant suppression of bone matrix deposition and bone mineralization. ${ }^{7}$ Furthermore, osteal macrophages located in the lining tissue of the bone surface support physiologic skeletal remodeling and anabolic actions of parathyroid hormone in bone. ${ }^{8}$ Depletion of myeloid lineage cells reduced cortical and trabecular bone mass and attenuated PTH-induced trabecular bone anabolism. Taken together, these results indicate that the macrophage function of clearing apoptotic cells in the bone marrow may consequently direct bone formation.

Within bone injury sites, cell-to-cell contact between macrophages and osteoblasts or bone marrow stromal cells

${ }^{1}$ Department of Periodontics and Oral Medicine, University of Michigan School of Dentistry, Ann Arbor, MI 48109, USA; ${ }^{2}$ Department of Periodontology, University of Florida College of Dentistry, Gainesville, FL 32610, USA and ${ }^{3}$ Department of Pathology, University of Michigan Medical School, Ann Arbor, MI 48109, USA

Correspondence: Laurie K McCauley (mccauley@umich.edu)

Received: 9 January 2015; Revised: 22 April 2015; Accepted: 23 April 2015 
(BMSCs) widely exists and is an essential step needed for macrophage efferocytosis of apoptotic cells. This kind of juxtacrine interaction may cause the juxtaposed cells to produce growth factors or pre-inflammatory cytokines, such as TGF- $\beta^{9}$ and IL- $6^{10}$ and may lead to chemotaxis migration effects on BMSCs in the surrounding environment.

It has been previously reported that the juxtacrine interaction of human myeloma-derived cells and BMSCs stimulated IL-6 secretion. ${ }^{11}$ Cytokine array data also identified a significantly higher level of IL-6 production from juxtacrine culture of macrophages and prostate cancer cells. ${ }^{12}$ IL-6 is a cytokine highly expressed in the bone marrow stroma and known for its role in bone homeostasis, ${ }^{13-15}$ such as maintaining the stemness of MSCs and accelerating cell migration and proliferation. ${ }^{10} \mathrm{~A}$ recent study reported that IL-6 enhanced the polarization of alternatively activated macrophages in order to resolve inflammation and improve wound healing. ${ }^{16}$ Furthermore, IL-6 signaling was found to play a vital role in bone anabolism. ${ }^{17}$ Thus, in this study, in order to explore the IL-6-mediated events in bone marrow triggered by the juxtacrine interaction of BMSCs, the effect of the juxtacrine interaction of mouse primary macrophages and BMSCs on IL-6 signaling and its effect on the migration and growth of BMSCs were assessed in vitro.

\section{MATERIALS AND METHODS}

Agents

Cell culture medium and reagents were purchased from Invitrogen. For western blots, the mouse $\beta$-Actin monoclonal antibody was from Sigma (St. Louis, MO), and mouse STAT3 and phospho-STAT3 monoclonal antibodies were from Cell Signaling (Boston, MA). The mouse IL-6 ELISA system, recombinant mouse IL-6, and human soluble glycoprotein protein 130 (sgp 130) were purchased from R\&D Systems (Minneapolis, MN).

\section{Cell culture}

BMSCs were flushed from femurs and tibiae of 4- to 8-weekold C57BL/6 mice (in-house bred; originally obtained from Jackson Labs, Bar Harbor, ME) and IL-6 KO mice (kindly provided by Evan Keller, University of Michigan, Ann Arbor, MI) and were incubated at $37^{\circ} \sim$ in $95 \% \mathrm{O}_{2} / 5 \% \mathrm{CO}_{2}$ in $\alpha-\mathrm{MEM}$ medium supplemented with $20 \%$ fetal bovine serum, $1 \%$ penicillin/streptomycin, 1\% glutamine (Invitrogen), and $10^{-8} \mathrm{~mol} \cdot \mathrm{L}^{-1}$ dexamethasone (Sigma). Bone marrow-derived macrophages were procured by culturing flushed bone marrow in $\alpha$-MEM medium containing $10 \%$ FBS, penicillin/streptomycin, and treating with M-CSF (30 ng. $\mathrm{mL}^{-1}$ ) (eBioscience, San Diego, CA) for 7 days.

Cell co-culture and preparation of conditioned medium To co-culture primary macrophages and BMSCs, $1 \times 10^{5}$ bone marrow-derived macrophages were seeded first into 6-well plates, cultured 24 hours, then $1 \times 10^{5}$ primary mouse BMSCs or apoptotic BMSCs (exposed to UV light treatment for 30 minutes) were placed on the macrophages in $\alpha$-MEM medium containing $10 \%$ FBS. The cocultures were harvested at different time points $(2,4,6$, 24 hours). At each time point, macrophages cultured alone, apoptotic BMSCs cultured alone, and BMSCs cultured alone were used as controls. The supernatants from all cultures were collected and stored at $-80^{\circ} \mathrm{C}$ until used as conditioned medium, or for IL-6 ELISA assay. Total RNA of cell cultures was harvested at 2 and 4 hours. Protein lysates of cell cultures were harvested at 6 and 24 hours.

\section{Scratch assay}

Primary mouse BMSCs were cultured in 24-well plates coated with collagen type I (Costar). Upon reaching confluence, the center of cell monolayers were scraped with a $200 \mu \mathrm{L}$ pipette tip. Cells were washed three times with PBS, then cultured with medium containing $1 \%$ serum and IL-6 protein ( $\left.10 \mathrm{ng} \cdot \mathrm{mL}^{-1}\right)$, or with different conditioned media for 8 hours. To determine whether the effect was gp130/IL-6/STAT-3 pathway dependent, the cells were pretreated with $1 \mu \mathrm{g} \cdot \mathrm{mL}^{-1} \mathrm{sgp} 130$ or vehicle control 1 hour before IL-6 treatment, or conditioned medium treatment. Photographs were taken of the cell cultures at and after cell scratching, and the width of gaps was measured by using the interactive measurement line tool. The fraction of initial defects after BMSC migration were calculated and compared among the different groups. Experiments were performed in duplicate.

In vitro BMSC migration assay

Migration assays were performed as described. ${ }^{9}$ with minor modifications. Briefly, cell migration was assessed in 24-well plate Transwells (Corning, Inc.) with a diameter of $6.5 \mathrm{~mm}$ and a pore size of $8 \mu \mathrm{m}$ coated with $0.5 \mu \mathrm{g} \cdot \mathrm{mL}^{-1}$ collagen type I (Millipore). BMSCs were placed in the upper chambers and conditioned media from co-culture, or $\alpha-M E M$ medium containing $10 \mathrm{ng} \cdot \mathrm{mL}^{-1} \mathrm{IL}-6$ protein and $1 \%$ FBS were added to the lower chambers. After 8 hours, the cells were fixed with $2.5 \%$ glutaraldehyde for 15 minutes, and the cells remaining on the top of the Transwell membranes were removed with cotton swabs. The cells that had migrated through the pores to the lower surface were stained with Gill's hematoxylin (Sigma-Aldrich). Four to five fields at $\times 200$ magnification were selected at random, photomicrographic images obtained, and the cells in each image counted. Experiments were performed in duplicate.

Mouse IL-6 ELISA assay

IL-6 protein levels in culture supernatants were measured with the Quantikine mouse IL-6 ELISA (R\&D systems) per manufacturer's instructions. Briefly, supernatant samples, 
standards, and controls were added to the anti-mouse IL-6 antibody pre-coated microplates and incubated at room temperature for 2 hours. Wells were washed extensively and then incubated with the IL-6 conjugate for another 2 hours. After extensive washing, the substrate solution was added and incubated for 30 minutes, followed by addition of the stop solution, and A450 values were measured using a microplate reader (Biochrom, Cambridge, UK).

Cell growth assay

BMSCs were plated at $1 \times 10^{5}$ cells per well in a 24-well plate for all groups of conditioned medium or $\alpha$-MEM medium containing $1 \%$ FBS supplied with $10 \mathrm{ng} \cdot \mathrm{mL}^{-1}$ mouse recombinant IL-6. For spg 130 inhibition, cells were pretreated with vehicle control (BSA) or sgp130 $\left(1 \mu \mathrm{g} \cdot \mathrm{mL}^{-1}\right)$ for 1 hour before adding IL-6. Growth of cells was assessed by hemacytometer counting at 1,2 , and 3 days after plating. For counting, each condition was plated in triplicate and duplicate counts of trypan blue negative cells per well were calculated (mean $\pm S E$ ).

Gene expression by real-time RT-PCR

Total RNA was isolated with TRIzol reagent (Invitrogen) from primary BMSCs and macrophages either alone or in co-culture after 2 and 4 hours. Reverse transcription PCR was conducted, and the cDNA products were amplified and detected using TaqMan Universal PCR master mix (Applied Biosystems, Foster City, CA, USA) and TaqMan probes, including mouse IL-6 (Mm03413826_mH), and mouse glyceraldehyde-3-phosphate dehydrogenase (GAPDH, Mm00446190) as an endogenous control. Realtime PCR was analyzed on ABI PRISM 7700 (Applied Biosystems, Foster City, CA, USA).

Western blot

After juxtacrine culture of mouse primary macrophages and BMSCs for 6 and 24 hours, cell lysates were prepared in lysis buffer containing 1\% Nonidet P-40, 5\% sodium deoxycholate, $1 \mathrm{mmol} \cdot \mathrm{L}^{-1}$ phenylmethylsulfonyl fluoride, $100 \mathrm{mmol} \cdot \mathrm{L}^{-1}$ sodium orthovanadate, and 1:100 protease inhibitor mixtures (Sigma). The protein concentration was measured using the Bio-Rad Protein detection system according to the manufacturer's instructions (Bio-Rad). About $15 \mu \mathrm{g}$ protein extracts were subjected to $10 \%$ SDS-PAGE and transferred to a polyvinylidene difluoride membrane (Bio-Rad) using a wet gel transfer system. The membranes were blocked with $5 \%$ nonfat milk 1 hour at $4^{\circ} \mathrm{C}$, then probed with the primary antibodies overnight at $4^{\circ} \mathrm{C}$. The immunocomplexes were visualized with horseradish peroxidase-coupled goat anti-rabbit or anti-mouse IgG (Promega) using the SuperSignal reagents (Pierce). The quantification of western blot signals was performed with Scionimage software $(\mathrm{NIH})$.
Statistical analyses

Statistical analyses were performed using unpaired t-tests. A P-value below 0.05 was considered statistically significant. One asterisk (*) represents a $P$-value below 0.05, two $\left({ }^{* *}\right)$ represent a $P$-value below 0.01. Data are presented as mean \pm SEM. All experiments were repeated a minimum of two times and representative assays presented.

\section{RESULTS}

Juxtacrine interaction of BMSCs and macrophages induced IL-6 activation

Our laboratory has previously shown by cytokine array that juxtacrine interaction of bone marrow-derived macrophages and high apoptotic prostate cancer cells significantly increased the presence of IL-6 proteins in the media after 24 hours of co-culture. ${ }^{12}$ We speculated that the increased IL-6 contributed to the polarization of macrophages into $M 2$ cells that aid in repair and resolution. Uchiyama reported higher IL-6 production when BMSCs were co-cultured with PU13 (human myeloid cancer cells). ${ }^{11}$ Thus, we hypothesized that juxtacrine culture of BMSCs and bone marrow-derived macrophages would also produce IL-6.

IL-6 mRNA levels in cells co-cultured for 2 or 4 hours were analyzed. In order to determine the effect of phagocytosis of apoptotic BMSCs by the macrophages on IL-6 mRNA levels, normal or apoptotic BMSCs were co-cultured with macrophages. To induce the apoptosis of BMSCs, cells were treated by UV light for 30 minutes, followed by incubation for another 2 hours. It was found that more than $90 \%$ of the cells were positive by trypan blue staining. Results from UV-treated BMSCs alone was not included in the Q-PCR experiment since the majority of cells were not alive. The Q-PCR results showed that the juxtacrine culture of macrophages and BMSCs expressed the highest levels of IL-6 mRNA, compared to either macrophages and BMSCs alone, or the juxtacrine culture of macrophages and apoptotic BMSCs (Figure 1a).

Over time, IL-6 protein levels in the supernatant from the BMSC and macrophage juxtacrine co-cultures were significantly higher at 2, 4, 6, and 24 hours evidenced by ELISA (Figure 1b). To further investigate the IL-6/STAT3 pathway activation in the cultures, the cell protein lysis was harvested at 6 hours of co-culture, and STAT3 phosphorylation evaluated by western blot. Juxtacrine culture of macrophages and BMSCs exhibited the highest P-STAT3 expression when compared to other groups (Figure $1 c$ and $d$ ).

Thus, the juxtacrine interaction of macrophages and live BMSCs significantly induced IL-6 signaling, while macrophage co-culture with apoptotic BMSCs did not induce IL-6 production. 

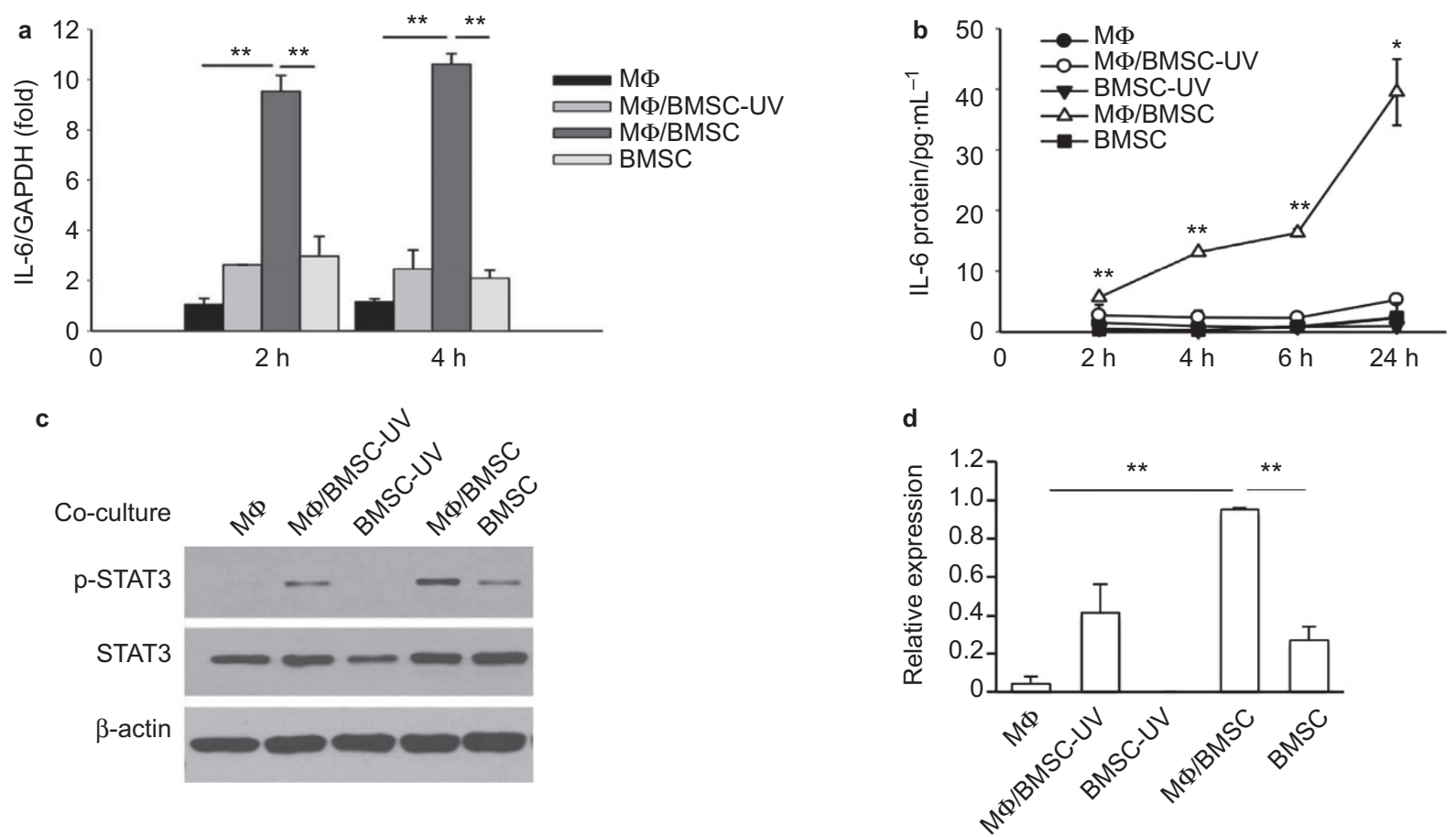

Figure 1. Juxtacrine interaction of BMSCs and macrophages (MФ) induced IL-6 activation. (a) Real-time PCR revealed significantly higher levels of IL-6 mRNA at 2 and 4 hours in co-cultures of mouse primary macrophages and BMSCs compared to co-cultures of macrophages and apoptotic BMSCs (UV treated), or macrophage and BMSC cultures alone. Data are mean $\pm \operatorname{SEM}(n=2$ in each group); $* * P<0.01$. (b) ELISA assay showed significantly higher levels of IL-6 protein in the supernatant from co-culture of mouse primary macrophages and BMSCs at 2, 4, 6, and 24 hours compared to co-culture of macrophages and apoptotic BMSCs, or macrophage and BMSC cultures alone. Data are mean \pm SE $(n=2$ in each group); $* P<0.05 ; * * P<0.01$. (c) Western blot analysis showed endogenous STAT3 phosphorylation was increased in co-cultures of primary macrophage and BMSCs after 6 hours when compared with either co-culture of macrophages and apoptotic BMSCs, or BMSCs, apoptotic BMSCs and macrophages alone. (d) Quantification for phosphorylated STAT3 protein expression relative to total STAT3. Data are mean \pm SEM of two independent experiments $(n=2$ in each group); $* * P<0.01$.

Conditioned media from juxtacrine cultures accelerated cell migration and BMSC numbers

The juxtacrine interaction of BMSCs and macrophages occurring in the bone marrow may affect the surrounding cells in the bone marrow environment by releasing vital signals. Since IL-6 was produced in juxtacrine cultures, and a recent study showed IL-6 promoted wound healing by stimulating the cell growth and migration of BMSCs, ${ }^{10}$ we questioned whether the juxtacrine signals of BMSCs and macrophages could indeed effect the migration and growth of BMSCs found in the local bone marrow environment. To investigate cell migration, we employed a Transwell assay of BMSCs (Figure 2a and b) and a cell scratch assay (Figure $2 \mathrm{c}$ and d). BMSCs were cultured for 8 hours in the different groups of conditioned medium. In both assays, conditioned medium from co-culture of BMSCs and macrophages significantly induced cell migration of BMSCs. While it has been shown that doubling of BMSC takes over 24 hours, we performed cell counts at this 8-hour time point to rule out the possibility of proliferation confounding the migration data. Cells were counted and no difference was found between treatment groups (Supplemental Figure 1a).
Additionally, the stimulation of conditioned media from BMSC and macrophage co-cultures, resulted in significantly higher BMSC numbers at 1,2 , and 3 days compared to control conditioned medium (Figure 2e). The dead cell ratio among the whole population was also measured by counting the positive cells via trypan blue staining, which showed no significant difference among the groups (data not shown). Thus, the conditioned medium from juxtacrine culture of BMSCs and macrophages induced cell migration and numbers of BMSCs.

Conditioned medium from IL-6 knockout $(\mathrm{KO})$ mouse cell cultures did not accelerate cell migration and BMSC cell numbers

In order to investigate the role of IL-6 signaling in the enhanced cell migration and growth of BMSCs induced by conditioned medium of juxtacrine BMSC and macrophage cultures, IL-6 ablated KO and wild-type (WT) mice were utilized to harvest BMSCs and macrophages in order to compare the effects of the conditioned medium of juxtacrine cultures with or without IL-6 protein on BMSCs.

Conditioned medium was collected from juxtacrine cultures as before from either IL-6 KO BMSCs and IL-6 KO macro- 


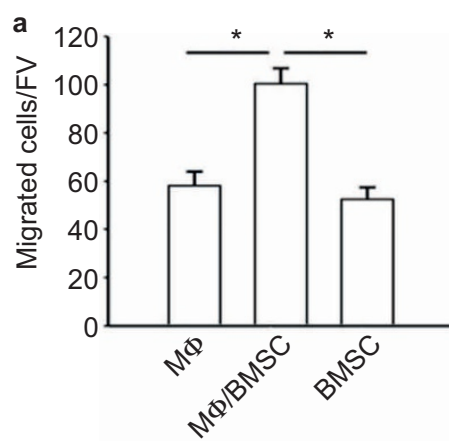

b

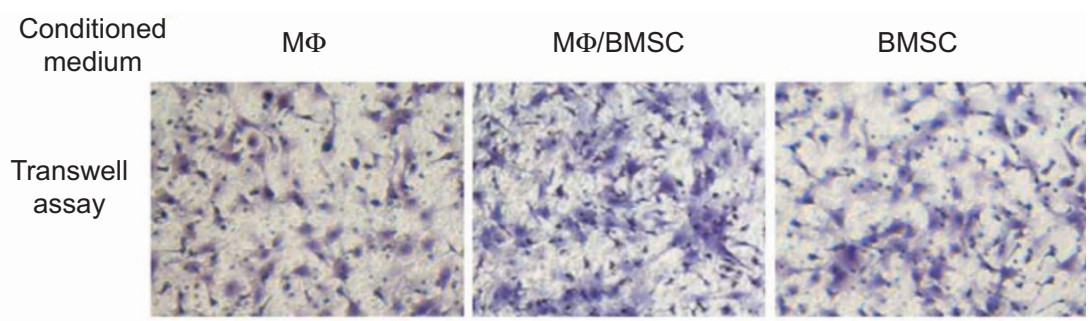

c
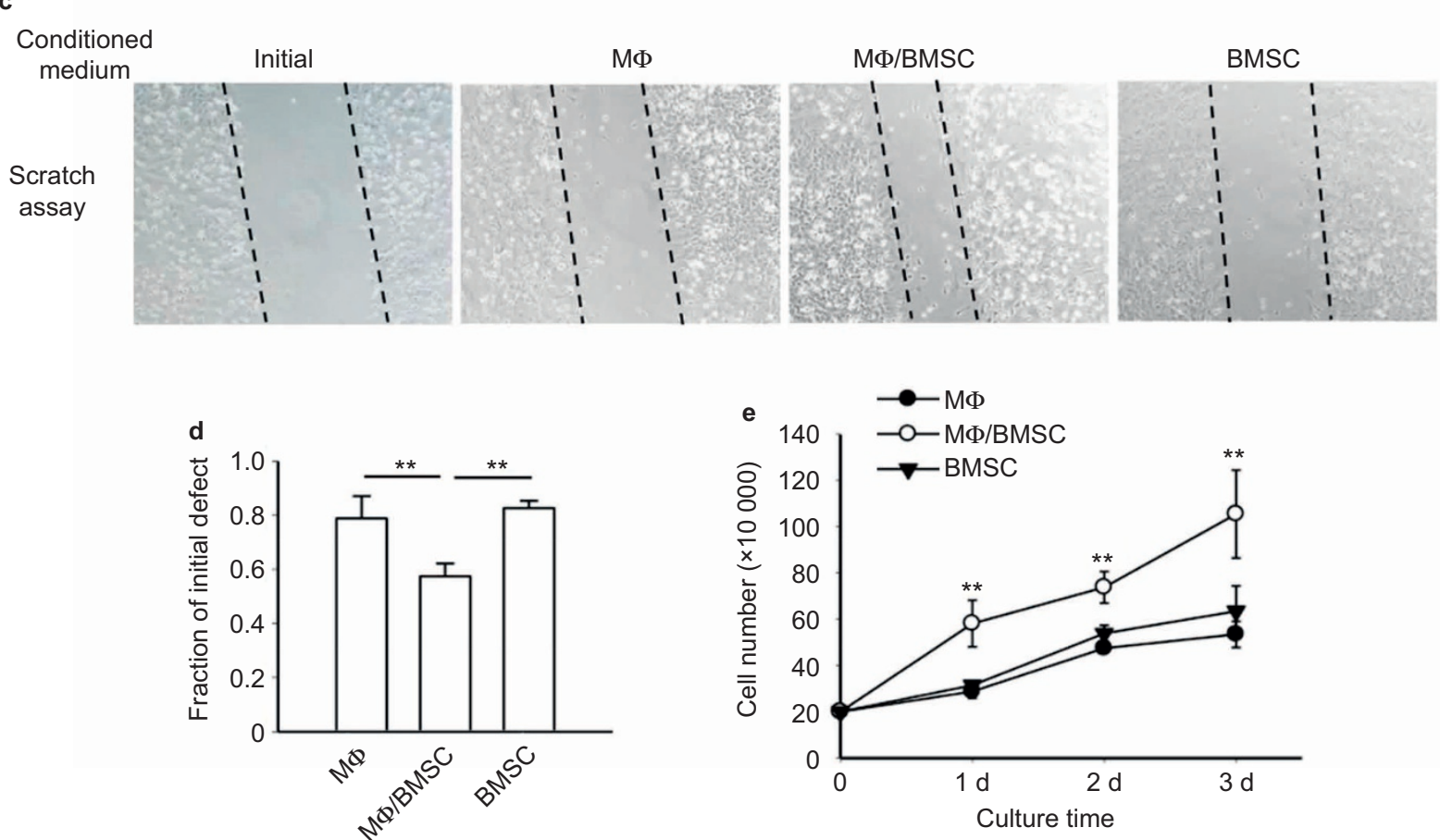

Figure 2. Conditioned media from juxtacrine culture accelerated cell migration and increased cell number of BMSCs. (a) Transwell migration assays of mouse BMSCs cultured for 8 hours in conditioned media from co-culture of macrophages and BMSCs, macrophage culture alone or BMSC culture alone. The migrated cells were counted after fixation and hematoxylin staining. Five fields of view (FV) were randomly picked up for each sample, and number of migrated cells per FV was counted. Quantitative results showed conditioned medium from co-culture of BMSCs and macrophages enhanced the migration of BMSCs. All experiments were performed in duplicate. Data are mean \pm SEM $(n=2$ in each group); $* P<0.05$. (b) Image of BMSCs after fixation and hematoxylin staining in the Transwell assays. Images were taken at $\times 200$ magnification. (c) Cell scratch assay for migration of mouse BMSCs cultured for 8 hours in conditioned media from co-culture of macrophages and BMSCs, macrophages cultured alone or BMSCs cultured alone. Cell scratches were imaged at the time of scratching (start point) and 8 hours after culture with conditioned medium. Images were taken at $\times 100$ magnification. (d) The remaining distance of scratch in each group was measured by its fraction of initial at the starting point. The remaining distance of scratch in BMSC cultures with conditioned media from co-culture of BMSCs and macrophages was significantly smaller than the other two groups. Data are mean \pm SEM $\left(n=3\right.$ in each group); ${ }^{* *} P<0.01$. (e) Conditioned medium from co-culture of BMSCs and macrophages increased mouse BMSCs after 1, 2, and 3 days of culture when compared to culture in conditioned medium from BMSCs or macrophages alone. Data are mean $\pm \operatorname{SEM}(n=3$ in each group); $* * P<0.01$.

phages or WT BMSCs and WT macrophages. Elevation of IL6 protein in the juxtacrine conditioned media from WT cells, and absence of IL-6 protein in the conditioned media from IL-6 KO cells was confirmed by ELISA assay (Figure 3a). Both groups of conditioned medium (from either WT or KO mice) were applied to the Transwell assays (Figure 3b) and cell scratch assays (Figure 3c) to compare their effects on cell migration of BMSCs. Both assays revealed that only the conditioned media generated from WT cells accelerated the migration of BMSCs, while the conditioned media from IL-6 KO cells was unable to elicit the same effect. Additionally, cell numbers from BMSC cultures were counted 1, 2, and 3 days after stimulation with the conditioned media from either WT cells or IL-6 KO cells, and the results showed only conditioned media from WT cells significantly increased cell numbers (Figure 3d). 

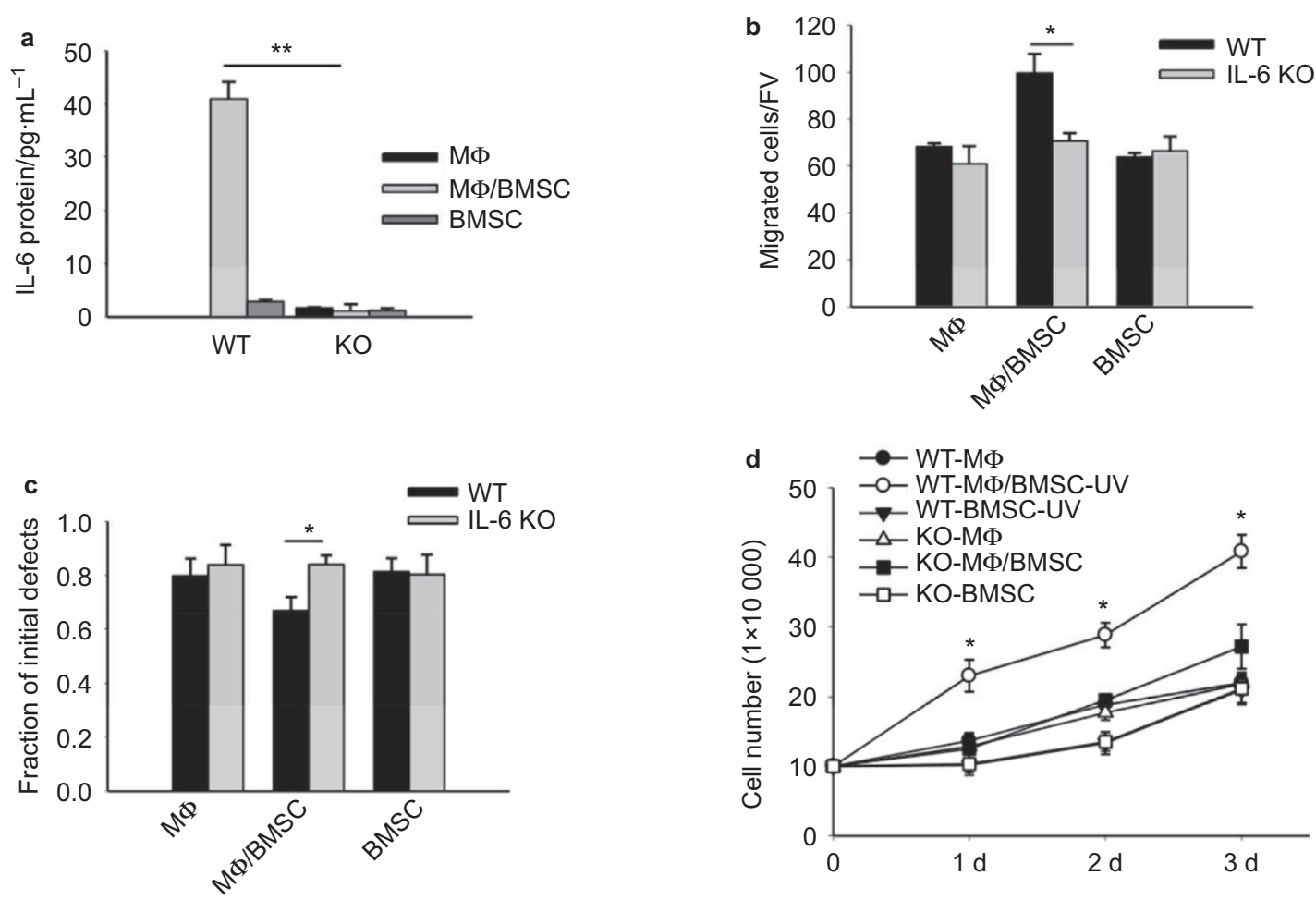

Figure 3. Conditioned medium from juxtacrine culture of IL-6 KO mice did not accelerate cell migration and cell numbers of BMSCs. (a) ELISA assay showed IL-6 protein significantly increased after 24 hours in the supernatant from co-culture of mouse macrophages and BMSCs from wildtype (WT) mice, while no IL-6 protein was secreted by the co-cultures from BMSCs and macrophages generated from IL-6 knockout (IL-6 KO) mice. Data are mean \pm SEM $\left(n=2\right.$ in each group); ${ }^{* *} P<0.01 ; * P<0.05$. (b) Transwell assays compared the migration of mouse BMSCs cultured for 8 hours in conditioned media from WT cells and in conditioned medium from IL-6 KO cells. Migrated cells were counted after fixation and hematoxylin staining. Five fields of view were randomly picked for each sample, and the number of migrated cells per field of view was counted. Only conditioned medium of co-cultures from WT cells increased migration of BMSCs. All experiments were performed in duplicate. Data are mean \pm SEM $(n=2$ in each group); $* P<0.05$. (c) Cell scratch assays compared the migration of mouse BMSCs cultured for 8 hours in conditioned media from WT cells or from IL-6 KO cells. Only conditioned medium of co-cultures from WT cells increased migration of BMSCs. All experiments were performed in duplicate. Data are mean \pm SEM $(n=3$ in each group); $* P<0.05$. (d) Only conditioned medium of co-culture from WT cells increased mouse BMSC numbers after 1,2 , and 3 days of culture. IL-6 KO groups did not affect cell number. Data were mean \pm SE $(n=3$ in each group); ${ }^{*} P<0.05$.

BMSCs are the main contributing cells of juxtacrine IL-6 production

A previous report suggested that human BMSCs were the primary cells to produce IL-6 protein when co-cultured with human myeloid cancer cells. ${ }^{1}$ However, the direct evidence for this speculation was not provided. In order to clarify whether it was BMSCs, macrophages, or both, which generate IL-6, co-cultures were established with WT-BMSCs and WT-macrophages, WT-BMSCs and IL-6 KO-macrophages, IL-6 KO-BMSCs and WT-macrophages, IL-6 KO-BMSCs and IL-6 KO-macrophages. The analysis of conditioned medium from these four groups and their single culture controls resulted in $\sim 50 \%$ reduction of IL-6 protein in WT-BMSC + IL-6 KO-macrophage cultures compared with co-cultures from WT cells. IL-6 KO in BMSCs demonstrated low levels of IL-6 protein in the conditioned media, regardless of the phenotype of macrophages (Figure 4).

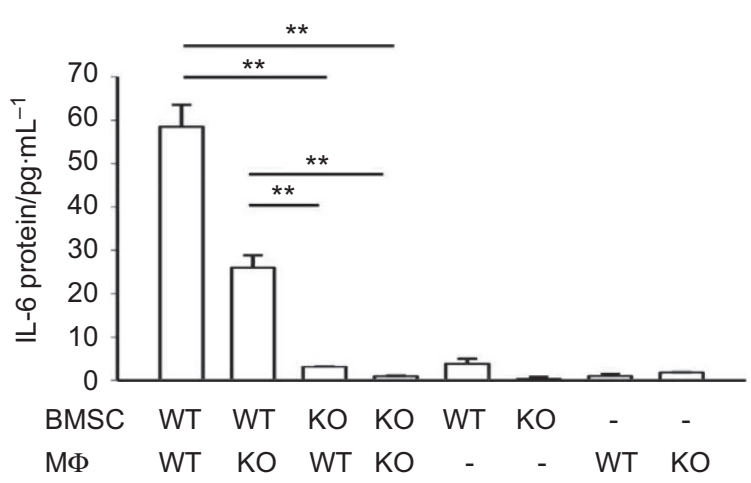

Figure 4. BMSCs were the main contributing cells for IL-6 protein production from juxtacrine interaction. ELISA assay showed that after 24 hours, IL-6 protein was significantly increased in the supernatant from co-culture of WT BMSCs and WT macrophages, and co-culture of WT BMSCs and IL-6 KO macrophages. No elevation of IL-6 protein concentration was found in the co-cultures of IL-6 KO BMSCs and either WT macrophages or IL-6 KO macrophages. Data are mean \pm SEM $(n=2$ in each group); $* * P<0.01$. 


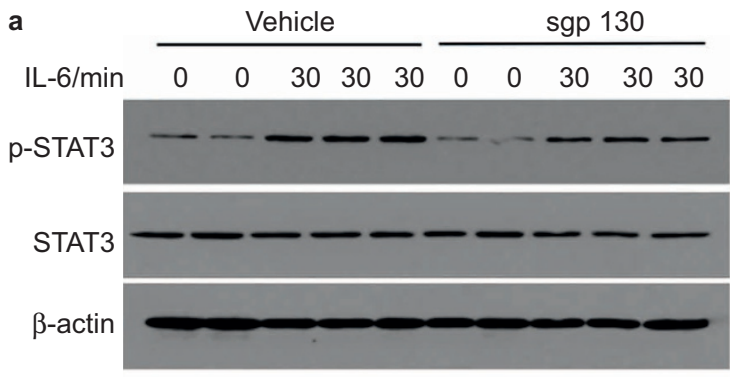

c
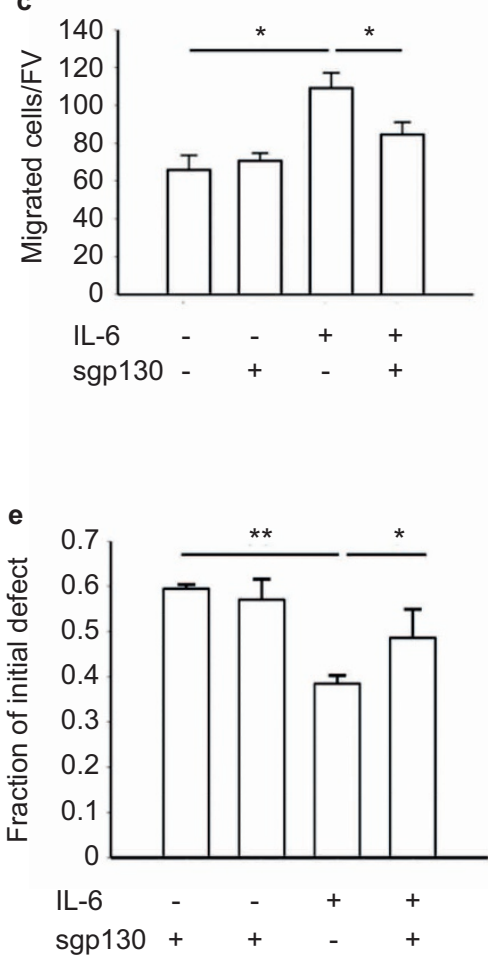

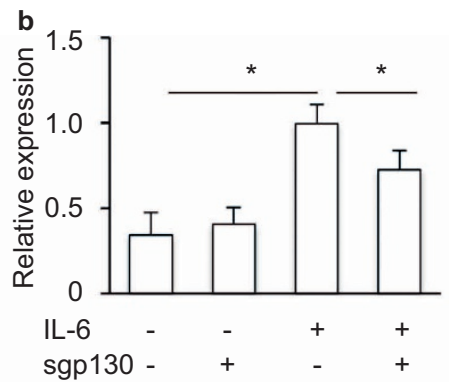

d

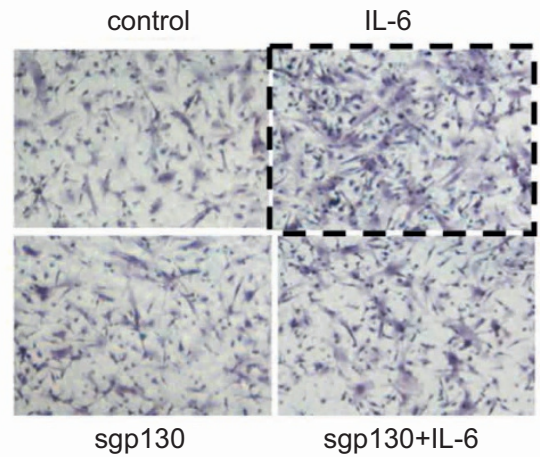

f

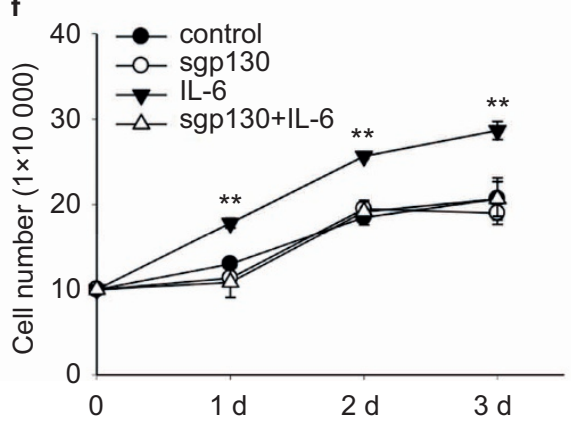

Figure 5. IL-6 protein accelerated cell migration and increased BMSC numbers through activation of the IL-6/gp130/STAT3 pathway. (a) BMSCs were treated with recombinant mouse IL-6 protein $\left(10 \mathrm{ng} \cdot \mathrm{mL}^{-1}\right)$ for 30 minutes with or without a 1-hour pretreatment of sgp130 $\left(1 \mu \mathrm{g} \cdot \mathrm{mL}{ }^{-1}\right)$. Western blot analysis of BMSC protein samples compared phosphorylation of STAT3 (pSTAT3). IL-6 protein stimulated the phosphorylation of STAT3 in BMSCs, which was blocked by spg130 pretreatment. (b) Quantification of p-STAT3 protein expression was determined relative to expression of STAT3. This experiment was performed in duplicate. Data are mean $\pm \mathrm{SEM} ; * P<0.05$. (c) Transwell assays compared the migration of BMSCs cultured for 8 hours after treatment with IL-6 protein $\left(10 \mathrm{ng} \cdot \mathrm{mL}^{-1}\right)$ with or without 1 -hour pretreatment of spg130 $\left(1 \mu \mathrm{g} \cdot \mathrm{mL}^{-1}\right)$. IL-6 protein treatment increased migration of BMSCs, while this effect was abolished by sgp130 pretreatment. The migrated cells were counted after fixation and hematoxylin staining. Images were taken at $\times 200$ magnification. Five fields of view (FV) were randomly picked for each sample, and number of migrated cells per FV was counted. All experiments were performed in duplicate. Data were mean \pm SEM $(n=2$ in each group); $* P<0.05$. (d) Image of BMSCs after fixation and hematoxylin staining in the Transwell assays. Images were taken at $\times 200$ magnification. (e) Cell scratch assays compared the remaining scratch distance of BMSC cultures after 8 hours of IL-6 protein $\left(10 \mathrm{ng} \cdot \mathrm{mL}^{-1}\right)$ treatment with or without pretreatment of spg130 $\left(1 \mu \mathrm{g} \cdot \mathrm{mL}{ }^{-1}\right)$. IL-6 protein treatment significantly reduced the remaining distance of cell scratch, which was abolished by sgp130 pretreatment. All experiments were performed in duplicate. Data are mean $\pm \operatorname{SEM}\left(n=3\right.$ in each group); $* P<0.05 ; * * P<0.01$. (f) IL-6 protein treatment $\left(10 \mathrm{ng} \cdot \mathrm{mL}^{-1}\right)$ increased BMSC numbers after 1,2 , and 3 days of culture, an effect which was abolished by spg130 pretreatment $\left(1 \mu \mathrm{g} \cdot \mathrm{mL}^{-1}\right.$ for 1 hours $)$. Data are mean $\pm \mathrm{SEM}(n=3$ in each group); $* * P<0.01$.

These results provided strong evidence that BMSCs were the major source of IL-6 in the juxtacrine cultures. When IL-6 production was ablated in BMSCs from IL-6 $\mathrm{KO}$ mice, the juxtacrine culture no longer induced IL-6 production. The experimental data from co-culture of macrophages with apoptotic BMSCs also supported this result. Once BMSCs were treated with UV light to induce their apoptosis, their co-culture with macrophages no longer produced IL-6 mRNA or protein (Figure $1 \mathrm{a}$ and $\mathrm{b}$ ). 
IL-6 protein accelerated cell migration and number by activation of IL-6/gp130/STAT3

In order to investigate downstream cell signaling, STAT3 phosphorylation was evaluated. For these studies, the soluble gp130 protein (sgp130), an inhibitor of the gp130/STAT3 pathway, was used to treat cells 1 hour before IL-6 stimulation. Western blot analyses demonstrated that after 30 minutes of treatment with IL-6, there was a significant increase in STAT3-phosphorylation which was blocked by the sgp 130 inhibitor (Figure $5 a$ and b).

When BMSCs were treated with IL-6 $\left(10 \mathrm{ng} \cdot \mathrm{mL}^{-1}\right)$ in the Transwell and cell scratch assays for 8 hours, or cultured in the dishes for 1, 2, and 3 days, IL-6 protein significantly increased BMSC migration (Figure $5 \mathrm{c}-\mathrm{e}$ ), and increased cell numbers (Figure 5f). Pretreatment of the cultures with sgpl30 (1 $\left.\mu \mathrm{g} \cdot \mathrm{mL}^{-1}\right) 1$ hour prior to IL-6 treatment attenuated the effect of IL- 6 treatment. Again, cell numbers were counted after 8 hours in the cell scratch assay and no difference was noted (Supplemental Figure 1b).

These data highlight that IL-6 protein accelerates cell migration and cell numbers of BMSCs by its activation of IL-6/gp130/STAT3 signaling pathway.

\section{Juxtacrine cultures accelerated BMSC migration and} numbers via IL-6/gp130/STAT3

The data presented above showed that the juxtacrine interaction of BMSCs and macrophages induced IL-6 which led to cell migration and increased BMSC numbers. To investigate the related underlying mechanism of this juxtacrine interaction, signaling pathways related to IL-6 in the co-cultures, such as STAT-3, SOCS-3, ERK, and NF-kB were investigated by looking at their activations triggered by juxtacrine interactions. The only pathway found activated via the juxtacrine interaction was STAT3 (p-STAT3 data presented in Figure 1, SOCS-3, ERK, and NF-KB not shown).

To further investigate the IL-6/STAT-3 pathway activation, BMSCs were directly treated with the conditioned medium from WT and IL-6 KO co-cultures to compare phosphorylation of STAT3 by western blot. The results showed that only the conditioned media from WT cell juxtacrine co-cultures could stimulate the phosphorylation of STAT3, while the conditioned media from IL-6 KO cell co-culture was unable to elicit the same effect (Figure $6 a$ and b). This indicated that lack of STAT3 activation in IL-6 KO juxtacrine conditioned media may contribute to its lack of ability to induce cell migration and increase cell numbers of BMSCs. To validate this finding, sgp130 was applied together with conditioned media from WT cell co-cultures, to evaluate its effect on cell migration and BMSC numbers. It clearly showed that, sgpl30 attenuated the cell migration (Figure 6c-e) and cell proliferation (Figure 6f) accelerated by the conditioned media from WT cell co-cultures.
Thus, the conditioned media from juxtacrine cultures accelerated cell migration and increased cell numbers by its activation of the IL-6/gp130/STAT3 pathway.

\section{DISCUSSION}

Little information is known as to how macrophages function in the bone microenvironment beyond their role as scavengers to cleanse the bone marrow of apoptotic cells. ${ }^{2}$ Recent studies from our laboratory and others have found that the macrophages located within bone marrow, exist as tissue resident macrophages involved in bone formation and mineralization in vitro and in vivo..$^{6-8}$ This phenomenon begs the question: What is the role of macrophages in the bone marrow in accelerating or maintaining bone formation? Since macrophages themselves do not produce bone matrix proteins, they must affect the function of either osteoblasts, which produce bone matrix, or their MSC precursors found in the bone microenvironment. To answer the question, we should not only look at the function of macrophages alone, but rather put them into the whole skeletal system and within the scope of osteoimmunology. Recently, studies have started to explore the interaction of immune cells with skeletal cells and to identify shared pathways and signaling molecules, in order to find osteoimmunological interactions in health and disease. ${ }^{18}$ Thus, in the present study, we investigated the function of macrophages in bone development by investigating their juxtacrine interaction with BMSCs. Central to our findings was the role of IL-6.

$\mathrm{IL}-6$ is a multifunctional cytokine that regulates various aspects of immune responses, acute-phase reactions and haematopoiesis. ${ }^{19}$ In the skeletal system, IL-6 is important in PTH induced bone resorption, ${ }^{20}$ is involved in osteoclast differentiation, ${ }^{21}$ and PTH induced support of hematopoietic progenitor cell expansion in bone marrow. ${ }^{22}$ IL-6 also stimulates osteoblastic proliferation through the gp130/STAT3 pathway, ${ }^{23}$ maintains MSC stemness and accelerates MSC migration and proliferation during wound healing. ${ }^{10}$ All of these findings indicate that IL-6 plays multiple functions in regulating bone metabolism and growth. Recently, other experiments in our lab showed a significant increase in the level of IL-6 protein found in the conditioned medium collected from co-culture of bone marrow macrophages and apoptotic prostate cancer cells, which may be linked to the polarization of macrophages induced by efferocytosis. ${ }^{12}$ Thus, we hypothesized that under physiological conditions, the juxtacrine interaction of macrophages and apoptotic BMSCs or osteoblasts occurring in bone turnover sites could induce IL-6 production, which would affect migration and growth of BMSCs from the local surrounding environment toward the turnover sites thus replacing the apoptotic cells phagocytosed by the 
a

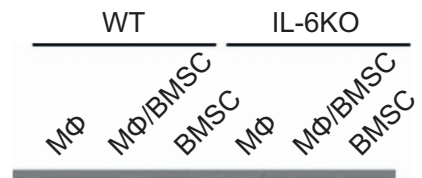

p-STAT3

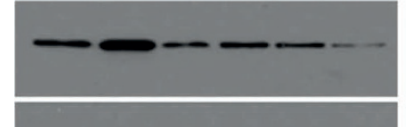

STAT3

$\beta$-actin

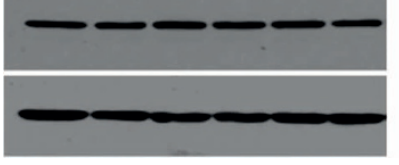

c
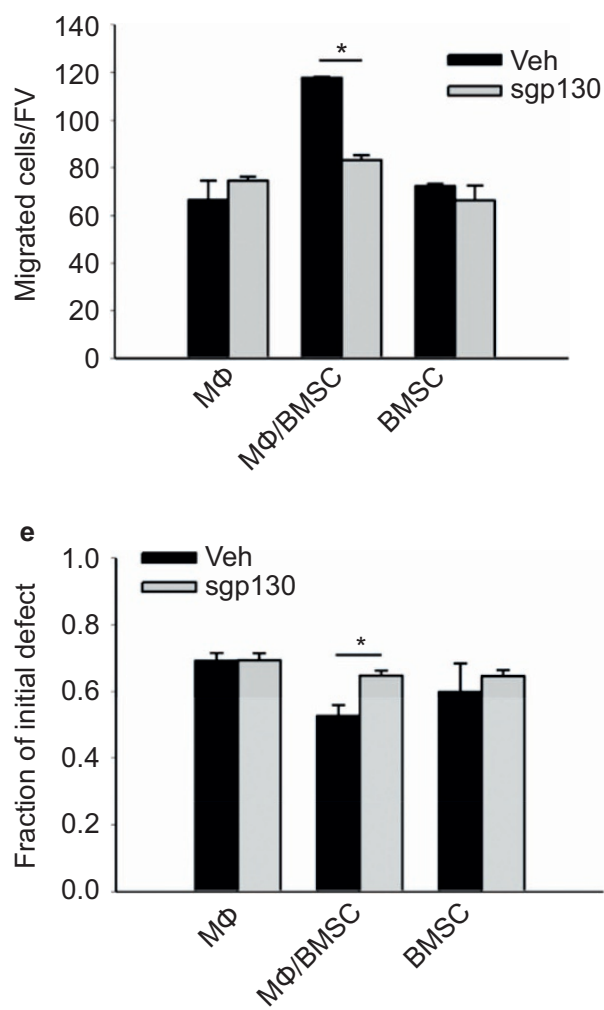

b

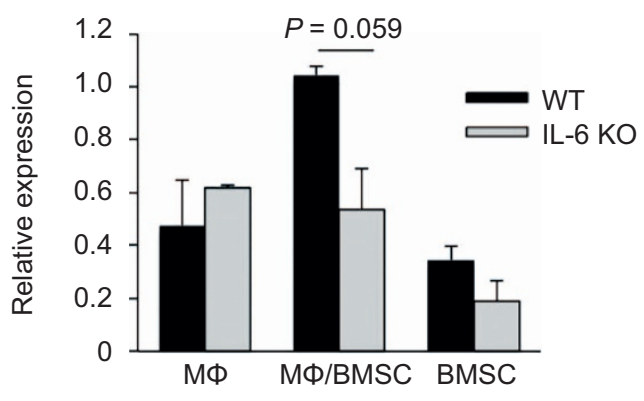

d

$\begin{array}{lll}\text { Conditioned M M M BMSC } & \text { BMSC }\end{array}$
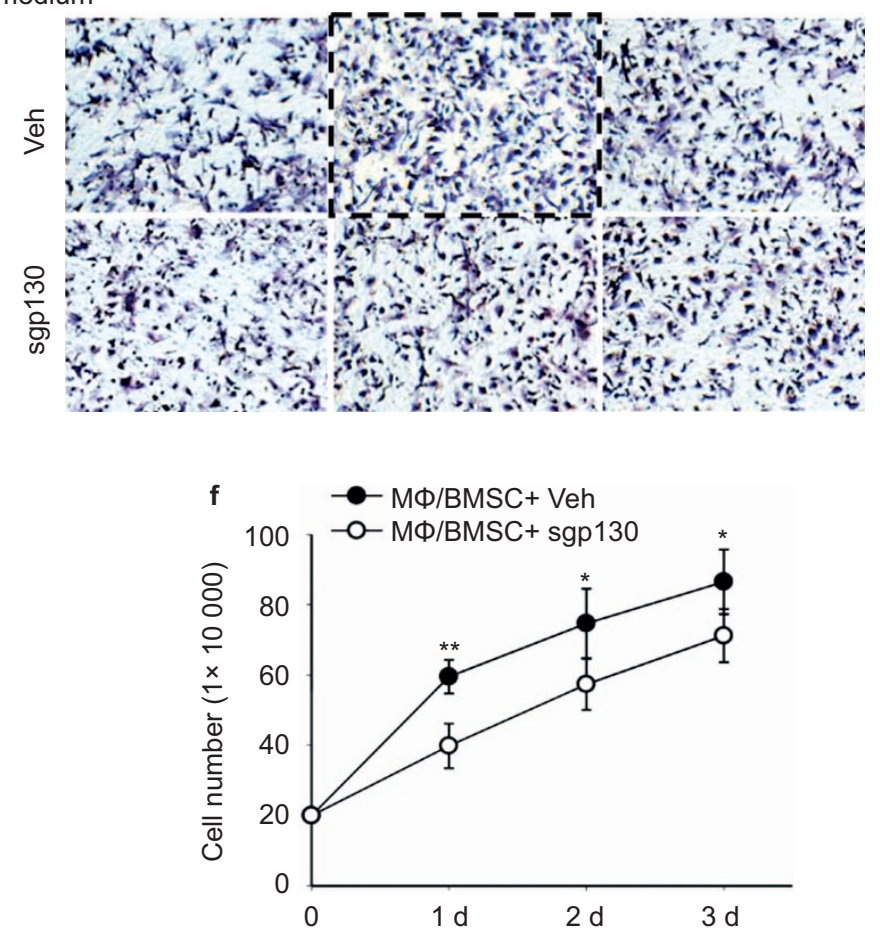

Figure 6. Juxtacrine interaction of BMSCs and macrophages accelerated BMSC migration and increased BMSC numbers through activation of the IL-6/ gp130/STAT3 pathway. (a and b) Conditioned medium harvested from either WT or IL-6 KO cell cultures were used to treat BMSCs for 30 minutes. Western blot analysis of BMSC proteins compared p-STAT3 levels. Only conditioned medium from co-culture of WT macrophages and BMSCs stimulated the phosphorylation of STAT3 in BMSCs. IL-6 KO conditioned medium did not. Quantification of p-STAT3 protein expression was determined relative to the expression of total STAT3. Data are mean \pm SEM $(n=2$ in each group); $P=0.059$. (c and d) Transwell assays compared the migration of mouse BMSCs cultured for 8 hours under the stimulation of conditioned medium from co-culture of WT BMSCs and macrophages with or without pretreatment of spg130 $\left(1 \mu \mathrm{g} \cdot \mathrm{mL}^{-1}\right)$. Pretreatment with sgp130 blocked the ability of the conditioned medium to stimulate BMSC migration. The migrated cells were counted after fixation and hematoxylin staining. Images were taken at $\times 200$ magnification. Five fields of view (FV) were randomly picked for each sample, and numbers of migrated cells per FV were counted. All experiments were performed in duplicate. Data were mean \pm SEM $(n=2$ in each group); $* P<0.05$. (e) Cell scratch assays compared the remaining scratch distance of BMSC cultures after 8 hours of conditioned medium from co-culture of WT BMSCs and macrophages with or without spg130 pretreatment $\left(1 \mu \mathrm{g} \cdot \mathrm{mL}^{-1}\right)$. Pretreatment with sgp130 blocked the stimulation of conditioned medium on the migration of BMSCs. All experiments were performed in duplicate. Data are mean \pm SEM $(n=3$ in each group); ${ }^{*} P<0.05$. (f) Pretreatment with spg130 $\left(1 \mu \mathrm{g} \cdot \mathrm{mL}^{-1}\right.$ for 1 hour) abolished the stimulation of conditioned medium on BMSC numbers after 1,2 , and 3 days of culture. Data are mean \pm SEM $(n=3$ in each group); $* P<0.05 ; * * P<0.01$.

macrophages, and hence contribute to bone formation and maintenance of bone homeostasis.

Experiments were performed to investigate the effect of juxtacrine interaction of macrophages and BMSCs on IL-6 expression by co-culturing primary bone marrow-derived macrophages and highly apoptotic BMSCs induced by UV light treatment. Concurrently, we also set up juxtacrine cultures of macrophages and normal BMSCs as a control 


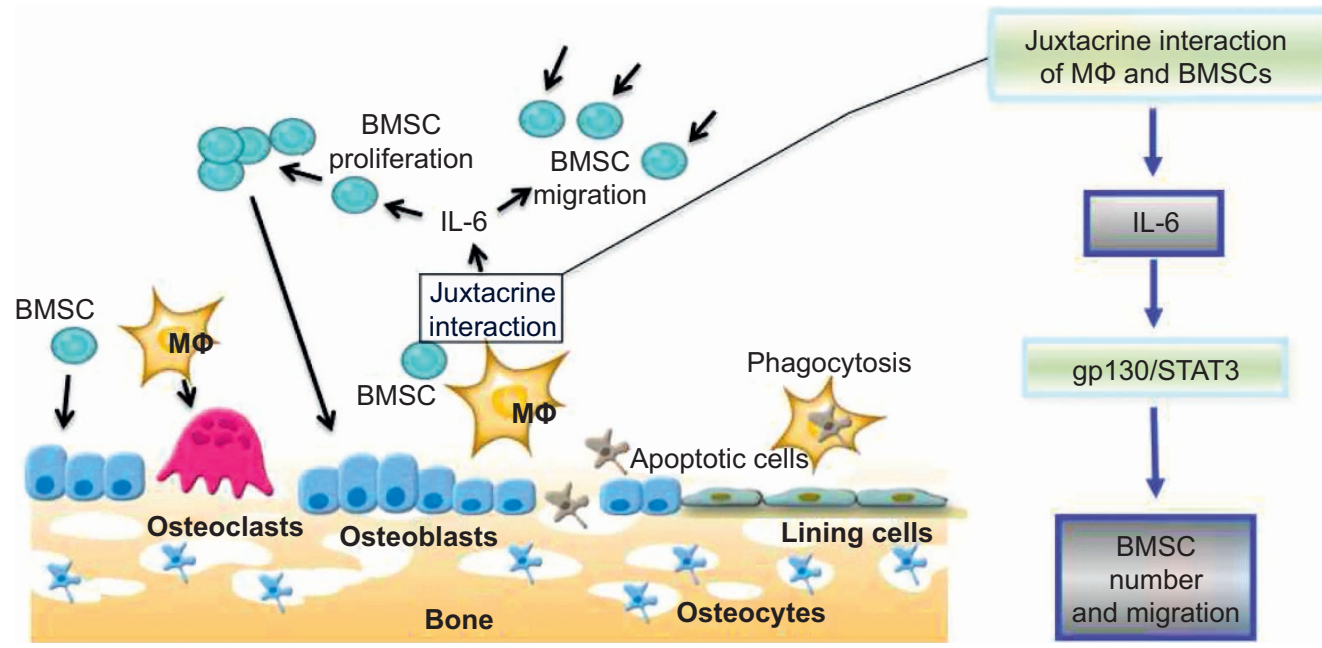

Figure 7. Overview of the mechanism of juxtacrine interactions of BMSCs and macrophages on BMSC migration and cell numbers. In the bone marrow environment, macrophages differentiate into osteoclasts and BMSCs differentiate into osteoblasts. The juxtacrine interaction of BMSCs and macrophages in the local bone marrow environment induced IL-6 production by BMSCs thus accelerating BMSC migration and increasing cell numbers. The juxtacrine interaction of BMSCs and macrophages accelerated BMSC migration and increased BMSC numbers through activation of the IL-6/gp130/ STAT3 pathway.

group to explore whether efferocytosis specifically alters IL-6 expression at the mRNA or protein level. Surprisingly, only juxtacrine cultures of live BMSCs and macrophages significantly supported IL-6 expression, while there was no increase of IL-6 expression in the juxtacrine cultures of apoptotic BMSCs and macrophages. When the conditioned media from co-cultures were used to investigate the migration and cell numbers of BMSCs, only the conditioned media from co-cultures of live BMSCs and macrophages showed significant changes, instead of the conditioned media from the co-culture of apoptotic BMSCs and macrophages. There are various possibilities to explain this. First, BMSCs are likely the primary cells to produce IL-6 triggered by the juxtacrine interaction. This hypothesis is supported by Uchiyama's study on juxtacrine culture of human myeloma-derived cell lines and BMSCs. In their study, significant IL-6 secretion was found in cocultures of a myeloma-derived cell line and BMSCs, however if the BMSCs were fixed with paraformaldehyde before co-culture, the IL-6 secretion was completely abrogated. " 1 Second, BMSCs and macrophages produce IL-6 after the trigger of juxtacrine interactions in a temporal manner. Analysis of the IL-6 protein in the conditioned media from co-cultures of combinations of WT- and IL-6 KO-BMSCs with WT- and IL-6 KO-macrophages, demonstrated that IL-6 expression by BMSCs is essential for the induction of IL-6 in macrophages during the juxtacrine interactions. When BMSCs from IL-6-ablated mice were used, the juxtacrine culture could no longer trigger the expression of IL- 6 protein. Indeed, when macrophages from IL-6-ablated mice were used, there was $\sim 50 \%$ reduction of IL-6 induction in the juxtacrine cultures. Thus, there may be a sequential effect of synthesis of IL-6 by the juxtacrine cultures in which BMSCs may be induced to produce IL-6 first. Therefore, when IL-6 can be normally synthesized in WT BMSCs and secreted outside the cells in a paracrine manner, it may stimulate macrophages to produce and secrete more IL-6 protein in both a paracrine manner and an autocrine feedback loop, which would explain the much higher total production of IL-6 seen in the whole WT co-cultures. When IL-6 was unable to be produced from the macrophages in the juxtacrine cultures, the whole amount of IL-6 secretion by the juxtacrine cultures was compromised. This experiment provides evidence to support that both BMSCs and macrophages are contributors for the production of IL-6 proteins in a temporal manner.

During the juxtacrine culture of macrophages and BMSCs, many cytokines and growth factors are secreted. In order to identify whether IL-6 was the specific inducer of migration and growth of BMSCs, we utilized IL-6 knockout mice to generate BMSCs and macrophages. By comparing the migration and cell growth of BMSCs stimulated with conditioned media from co-cultures of WT cells and IL-6 $\mathrm{KO}$ cells, it was found that only conditioned media containing IL-6 stimulated the migration and increased the cell numbers of BMSCs, while conditioned media lacking IL-6 could not. However, the conditioned media from co-culture of macrophages and apoptotic BMSCs did show a trend of increasing migration of BMSCs, although its IL-6 protein levels were as low as those from BMSC cultures and macrophage cultures alone (data not shown). Therefore, we cannot completely exclude other growth factors and cytokines that may also play a role. 
Thus, further experiments, such as gene comparisons, would definitely provide a more comprehensive profile for evaluation.

In the present study, STAT3 was found to be activated in BMSCs by stimulation of either exogenous IL-6 protein, or the conditioned media from co-cultured macrophages and BMSCS. STAT3 is the downstream regulator of $\mathrm{gp} 130 /$ IL-6 pathway, and its activation can be blocked by soluble gp 130. ${ }^{13,24}$ To investigate whether conditioned media or IL-6 protein enhances migration and growth of BMSCs via their activation of the gp130/STAT3 pathway, soluble gp130 (sgp 130) was utilized in the cell migration and cell number assays. The data showed that once gp130/STAT3 activation in BMSCs was blocked by sgp 130, the increase in migration and cell numbers of BMSCs by IL-6 protein or conditioned media treatment was abolished. Co-cultures of BMSCs and macrophages also exhibited the highest level of STAT3 phosphorylation, which is consistent with the change in IL-6 protein level. This means that the IL-6/ STAT3 pathway in the co-cultures was activated by the IL-6 produced and secreted into the conditioned media, suggesting a positive autocrine feedback loop in such a coculture environment which may function to augment IL-6 signals to neighboring BMSCs.

In summary, we found that juxtacrine interactions of BMSCs and macrophages alters the biological function of BMSCs through the IL-6/gp130/STAT3 pathway. Our laboratory previously reported that sIL-6R upregulated myeloid cell expansion and production of transforming growth factor- $\beta$ (TGF- $\beta$ ) in the bone microenvironment. ${ }^{17}$ A next step would be to examine the effect of this juxtacrine interaction on SIL-6R expression and its effects on myeloid cell expansion. Indeed, while our current study focused on the function of juxtacrine interaction of macrophages and BMSCs from mice under physiologic conditions, it would be interesting to investigate the juxtacrine relationship under certain disease models such as bone fracture healing or in the context of age related osteoporosis.

Juxtacrine interactions of BMSCs and macrophages activated the secretion of IL-6 into co-culture supernatants that accelerated cell migration and increased BMSC numbers via activation of the IL-6/gp130/STAT3 pathway in BMSCs. While BMSCs in the juxtacrine cultures were the primary contributor for the secretion of IL-6, macrophages did contribute to the overall production of IL-6 (Figure 7). These findings are important for understanding the function of macrophages in bone formation by effecting the migration and growth of BMSCs in the bone microenvironment.

\section{Competing interests}

The authors declare no conflict of interest.

\section{Acknowledgements}

This work was supported by National Institutes of Health Grants DK053904 and CA093900 (to Laurie K. McCauley).

\section{References}

1 Pittenger MF, Mackay AM, Beck SC et al. Multilineage potential of adult human mesenchymal stem cells. Science 1999; 284:143-147.

2 Murray PJ, Wynn TA. Protective and pathogenic functions of macrophage subsets. Nat Rev Immunol 2011; 11: 723-737.

3 McCauley LK, Dalli J, Koh AJ, Chiang N, Serhan CN. Cutting edge: parathyroid hormone facilitates macrophage efferocytosis in bone marrow via proresolving mediators resolvin D1 and resolvin D2. J Immunol 2014; 193: 26-29.

$4 \mathrm{Wu}$ AC, Raggatt LJ, Alexander KA, Pettit AR. Unraveling macrophage contributions to bone repair. Bonekey Rep 2013; 2:373.

5 Chang MK, Raggatt LJ, Alexander KA et al. Osteal tissue macrophages are intercalated throughout human and mouse bone lining tissues and regulate osteoblast function in vitro and in vivo. J Immunol 2008; 181: 1232-1244.

6 Pettit AR, Chang MK, Hume DA, Raggatt LJ. Osteal macrophages: a new twist on coupling during bone dynamics. Bone 2008; 43: 976-982.

7 Alexander KA, Chang MK, Maylin ER et al. Osteal macrophages promote in vivo intramembranous bone healing in a mouse tibial injury model. J Bone Miner Res 2011; 26: 1517-1532.

8 Cho SW, Soki FN, Koh AJ et al. Osteal macrophages support physiologic skeletal remodeling and anabolic actions of parathyroid hormone in bone. Proc Natl Acad Sci U S A 2014; 111: 1545-1550.

9 Tang Y, Wu X, Lei W et al. TGF-beta1-induced migration of bone mesenchymal stem cells couples bone resorption with formation. Nat Med 2009; 15: 757-765.

10 Pricola KL, Kuhn NZ, Haleem-Smith H, Song Y, Tuan RS. Interleukin-6 maintains bone marrow-derived mesenchymal stem cell stemness by an ERK1/2-dependent mechanism. J Cell Biochem 2009; 108: 577-588.

11 Uchiyama H, Barut BA, Mohrbacher AF, Chauhan D, Anderson KC. Adhesion of human myeloma-derived cell lines to bone marrow stromal cells stimulates interleukin-6 secretion. Blood 1993; 82: 3712-3720.

12 Soki FN, Koh AJ, Jones JD et al. Polarization of prostate cancer associated macrophages is induced by milk-fat globule-EGF factor 8 (MFG-E8)mediated efferocytosis. J Biol Chem 2014; 289: 24560-24572.

13 Ogasawara H, Tsuji T, Hirano D, Aoki Y, Nakamura M, Kodama H. Induction of IL-6 production by bone marrow stromal cells on the adhesion of IL-6-dependent hematopoietic cells. J Cell Physiol 1996; 169: 209-216.

14 Manolagas SC. Role of cytokines in bone resorption. Bone 1995; 17: 63S-67S.

15 Manolagas SC, Jilka RL. Bone marrow, cytokines, and bone remodeling. Emerging insights into the pathophysiology of osteoporosis. N Engl J Med 1995; 332: 305-311.

16 Fernando MR, Reyes JL, Iannuzzi J, Leung G, McKay DM. The proinflammatory cytokine, interleukin-6, enhances the polarization of alternatively activated macrophages. PLoS One 2014; 9: e94188.

17 Cho SW, Pirih FQ, Koh AJ et al. The soluble interleukin-6 receptor is a mediator of hematopoietic and skeletal actions of parathyroid hormone. J Biol Chem 2013; 288: 6814-6825.

18 McHugh KP. Osteoimmunology in skeletal cell biology and disease. Autoimmunity 2008; 41: 181-182.

19 Kopf M, Baumann H, Freer G et al. Impaired immune and acute-phase responses in interleukin-6-deficient mice. Nature 1994; 368: 339-342.

20 Grey A, Mitnick MA, Masiukiewicz U et al. A role for interleukin-6 in parathyroid hormone-induced bone resorption in vivo. Endocrinology 1999; 140: 4683-4690 
21 Palmqvist P, Persson E, Conaway HH, Lerner UH. IL-6, leukemia inhibitory factor, and oncostatin $\mathrm{M}$ stimulate bone resorption and regulate the expression of receptor activator of NF-kappa B ligand, osteoprotegerin, and receptor activator of NF-kappa B in mouse calvariae. J Immunol 2002; 169: 3353-3362.

22 Pirih FQ, Michalski MN, Cho SW et al. Parathyroid hormone mediates hematopoietic cell expansion through interleukin-6. PLoS One 2010; 5: e13657.

23 Sims NA, Jenkins BJ, Quinn JM et al. Glycoprotein 130 regulates bone turnover and bone size by distinct downstream signaling pathways. $J$ Clin Invest 2004; 113: 379-389.
24 Garbers C, Thaiss W, Jones GW et al. Inhibition of classic signaling is a novel function of soluble glycoprotein 130 (sgp130), which is controlled by the ratio of interleukin 6 and soluble interleukin 6 receptor. J Biol Chem 2011; 286: 42959-42970.

(1) This work is licensed under a Creative Commons Attribution(c) Non Commercial-NoDerivs 3.0 Unported License. The images or other third party material in this article are included in the article's Creative Commons license, unless indicated otherwise in the credit line; if the material is not included under the Creative Commons license, users will need to obtain permission from the license holder to reproduce the material. To view a copy of this license, visit http://creativecommons.org/licenses/by-nc-nd/3.0/

Supplemental Information for this article can be found on the Bone Research website (http://www.nature.com/boneres). 